\title{
Coarse Irradiance Estimation using Curvilinear Skeleton
}

\author{
Laurent Noël* \\ UPE MLV, LIGM
}

\author{
John Chaussard ${ }^{\dagger}$ \\ UPE MLV, LIGM
}

\author{
Venceslas Biri \\ UPE MLV, LIGM
}
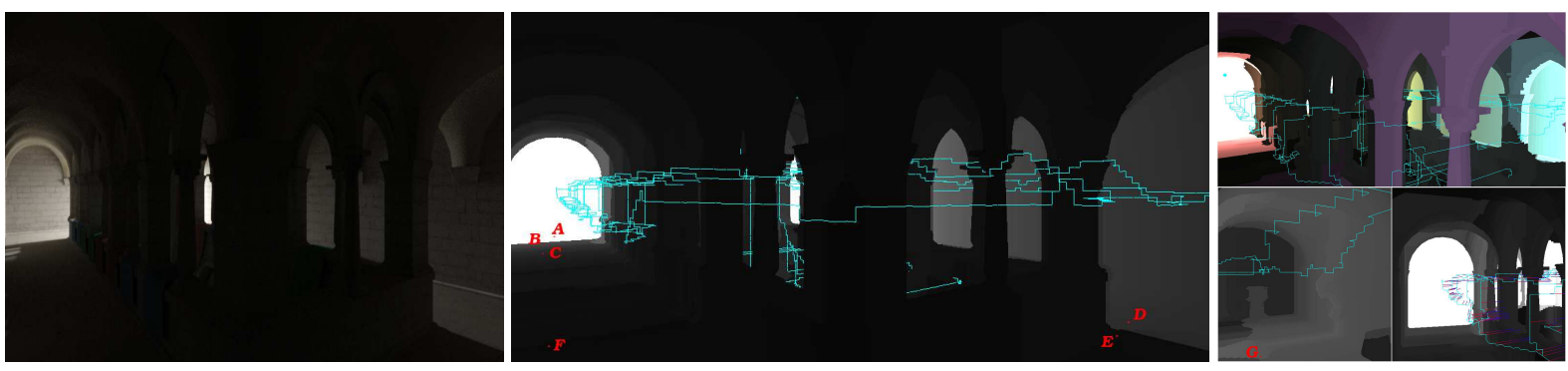

Figure 1: Left. Ground truth image. Center : backward irradiance estimation and the skeleton, red points are used for comparison. Right : several screenshot showing direction, a different point of view in the scene and an exemple with multiple lights

Keywords: global illumination, irradiance, skeletonization

\section{Introduction}

Light flux can take complex paths to reach the observer, especially in large and complex scenes. In such situations, global illumination algorithms are used : irradiance caching, path tracing and radiosity allow to precisely compute the irradiance at any point of the scene, but remain quite slow. Our present work aims to evaluate, very coarsely, the irradiance of any point of a scene, allowing to give, to global illumination algorithms, information about the main direction and intensity of the light flux. We use a coarse representation of the scene using a skeleton of its voids (where the light propagates). We present two heuristics to compute a coarse radiance estimation and the main light stream orientation. Our work offers the possibility to define many other heuristics.

\section{Coarse irradiance estimation}

Step 1 - Voxelization In this stage, we compute the voxelization of the scene. We can use any real-time voxelization algorithm such as [Eisemann and Décoret 2008]. Moreover, after the skeletonization process (see next paragraph), we compute, for each voxel of the scene, its closest skeleton node.

Step 2 - Skeletonization We compute the curvilinear skeleton using a novel approach based on cubical complexes. The skeleton is automatically filtered using the distance map and the opening function of the discrete scene, and a saliency information computed on the skeleton's points. The result is a pruned skeleton (no extra-elements due to noise) with the same "visual aspect" than the scene. Moreover, the skeleton is a one-dimensional object which can be seen as a graph. We then compute the shortest path, along the skeleton, between any skeleton's node and any scene's light.

Step 3 - Heuristics for irradiance computation We present two simple heuristics to estimate both radiance and main light stream orientation : a backward and a forward estimation. The backward estimation starts from a node $n_{c}$, and seeks the farthest visible node $n_{e}$ along the shortest path, on the skeleton, towards light $l$. We then compute the distance $d_{\text {all }}=d\left(l, n_{e}\right)+d\left(n_{c}, n_{e}\right)$ to obtain the final

\footnotetext{
*e-mail: lnoel@etudiant.univ-mlv.fr

†e-mail: chaussaj@esiee.fr
}

\begin{tabular}{|l|c|c|c|c|c|c|c|}
\hline Point & A & B & C & D & E & F & G \\
\hline Reference & 1.05 & 0.23 & 0.10 & 0.08 & 0.008 & 0.01 & $4 \mathrm{e}-3$ \\
\hline backward & 1.17 & 0.26 & 0.14 & 0.17 & 0.036 & 0.04 & 0.03 \\
\hline forward & 1.17 & 0.22 & 0.10 & 0.01 & 0.005 & $9 \mathrm{e}-3$ & 0.01 \\
\hline
\end{tabular}

Table 1: Irradiance $\left(\mathrm{W} / \mathrm{m}^{2}\right)$ computed in several scene points

irradiance $B=L_{0} /\left(\pi * d_{\text {all }}^{2}\right), L_{0}$ being the radiance of the source. The forward estimation dispatches the flux of the light along the skeleton, using a breadth first search starting from the light's skeleton node. The flux arriving at a node is multiplied by a factor depending on the relative size of the maximal balls between adjacent nodes. This flux is divided by the square of the total distance along the skeleton to give the final irradiance.

\section{Results and discussion}

We implemented a simple openGL visualization of our heuristics as shown in figure 1. Our evaluation (part 3) took an average of $3.5 \mathrm{~s}$ per light for the backward heuristic and $812 \mathrm{~ms}$ for the forward one (using a $2.26 \mathrm{GHz}$ P8400 with 3GB RAM). The voxelization costs $123 \mathrm{MB}$ but the skeleton is only $1.56 \mathrm{MB}$. We will provide an open source OpenGL/GLSL implementation of both heuristics.

We compare our estimation with a traditional path tracer (we use Embree from Intel) in various points, directly lighted or not. Both heuristics give a coarse estimation of radiance since the reflectivity of surface is considered as homogeneous, and more importantly, any node can perform a diffuse reflection in any direction even if its border is empty. However, results show that our evaluation diverge only in very dark areas mainly due to the last mentionned limitation. We believe that such information can be valuable especially for global illumination algorithms. Note that backward heuristic can be used as an upper bound to irradiance estimation. The estimation, on each skeleton node, of an average oriented reflectivity will improve the correctness of our method. Our work is also designed to accept new heuristics.

\section{References}

Eisemann, E., AND DÉCORET, X. 2008. Single-pass GPU Solid Voxelization and Applications. In GI '08: Proceedings of Graphics Interface 2008, vol. 322, 73-80. 